Portland State University

PDXScholar

6-17-2019

\title{
Parasocial Relationships with Podcast Hosts
}

Mikhaela Nadora

Portland State University

Follow this and additional works at: https://pdxscholar.library.pdx.edu/honorstheses Let us know how access to this document benefits you.

\section{Recommended Citation}

Nadora, Mikhaela, "Parasocial Relationships with Podcast Hosts" (2019). University Honors Theses. Paper 771.

https://doi.org/10.15760/honors.789

This Thesis is brought to you for free and open access. It has been accepted for inclusion in University Honors Theses by an authorized administrator of PDXScholar. Please contact us if we can make this document more accessible: pdxscholar@pdx.edu. 


\title{
Parasocial Relationships with Podcast Hosts
}

by

Mikhaela Nadora

An undergraduate honors thesis submitted in partial fulfillment of the requirements for the degree of

\author{
Bachelor of Arts \\ in \\ University Honors \\ and \\ Communication Studies
}

Thesis Adviser

Dr. Lee Shaker

Portland State University 


\begin{abstract}
This study is a deductive thematic analysis of podcast hosts' attributes that explores how parasocial relationships (PSR) might be cultivated with listeners. I analyze five episodes of one podcast, Stuff You Should Know and use its transcriptions to code for themes of identification, conversation practices, and authenticity to suggest ways PSR is developed by the podcast's hosts.
\end{abstract}

Results found host behaviors suggest how listeners may react to them. The combination of the effect of social deixis while describing personal characteristics suggest listeners may feel physically and emotionally closer to the hosts. Listeners may also see them as more trustworthy because the hosts care for listeners' wellbeing. Lastly, when hosts respond to the parasocial conversation, listeners may feel their effort to the PSR is reciprocated. In all, this study suggests that podcast hosts' behaviors contribute to listeners' PSR.

There are now ways to personalize our viewing and listening experiences to cater to our interests. Because of this, relationships with media figures may become stronger than ever. If this is the case, we must continue to research the links between media, media technology, and media consumers, in order to adapt to this new media landscape. Podcasts are especially applicable to these circumstances. 


\section{Introduction}

This study is a deductive thematic analysis of podcast hosts' attributes that explores how parasocial relationships (PSR) might be cultivated with listeners. I analyze five episodes of one podcast, Stuff You Should Know. I use the podcast's transcriptions to code for themes of identification, conversation practices, and authenticity to suggest ways PSR is developed by the podcast's hosts.

Results found certain repeated behaviors by the hosts that suggest how listeners may react to those behaviors. The combination of the effect of social deixis while describing personal characteristics suggest listeners may feel physically and emotionally closer to the hosts. Listeners may also see them as more trustworthy because the hosts care for listeners' wellbeing and how to educate them well. Lastly, when hosts respond to the parasocial conversation, listeners may feel their effort to the PSR is reciprocated. In all, this study suggests that podcast hosts' behaviors contribute to listeners' parasocial interactions (PSI) and its culmination over time results into a PSR.

The importance of this study regards how we consume media. There are now ways to personalize our viewing and listening experiences to cater to our interests. Because of this, relationships with media figures may become stronger than ever. If this is the case, we must continue to research the links between media, media technology, and media consumers, in order to adapt to this new media landscape. Podcasts are especially applicable to these circumstances.

\section{Parasocial Interaction}

Horton and Wohl (1956), the creators of parasocial interaction theory (PSI) define it as:

“one-sided, nondialectical, controlled by the performer, and not susceptible of mutual development" (p. 215). This non-reciprocated phenomenon is thought of as an "illusion of intimacy" (p. 217) which is only experienced by the audience. 
A "persona" (p. 216) is the term used in the article to describe the media figure one has a PSI with. The audience has free will to choose only out of the personae offered, as they cannot singlehandedly create these relationships. As an extension of PSI, a PSR is defined by Horton and Wohl as: the accumulation of perceived knowledge one has of a persona, the culmination of past experiences concerning them, and ongoing PSI with them. The original study applied the aspects of PSI and PSR to game show personalities and radio hosts. In the end, they propose to extend their newly found conjectures to other forms of mass media.

\section{Functionality and Technical Traits of Podcasts}

Podcasts are the latest advancement in auditory media. Perhaps its largest difference between radio and podcasts lies in their respective distribution processes. Anyone can create a podcast: it is free, there is no license to get beforehand, no media gate-keepers, and it can be done in the comfort of a "makeshift studio" (Berry, 2016, p. 14). The "de-professionalization" (Berry, 2016, p. 14) of podcast production allows for podcast hosts to "have more freedom to be casual, creative, and experimental" (McHugh, 2016, p. 70), because they are in control of their content and understand what their listeners want from them. Although podcasting is not controlled by corporations the way radio stations are, they are starting to be grouped or owned by podcast networks (Hilmes \& Lindgren, 2016). Many podcasts, however, are not financed by these networks. Instead, they get their funding from sponsors (McHugh, 2016). In return, hosts promote a product or service for them in each episode during advertisement breaks. Even though ads are voiced by the hosts themselves, they and their listeners are aware of, tolerate, and understand it is necessary, in order to keep the podcast in business (McClung \& Johnson, 2010; McHugh, 2016). The freedom to create through the de-professionalized functional and technical traits of podcasts becomes complicated when hosts must consider the interests of their listeners. 
A host has certain freedoms when producing a podcast, but the job also has its pressures. For one, even though PSRs are not reciprocated yet hosts still have a sense of their demographic. Markman and Sawyer (2014) found that podcast hosts feel they are contributing to a community of their same interests and try to be inclusive of topics and audiences that are not usually addressed by the radio. The same research sought to learn what motivates podcast hosts to create content. According to the study, motivations include: interest in the technology of the medium, self-expression, attention seeking, and promoting other content and/or people. Although, hosts feel they must be constantly engaged with the content and their imaginary audience when recording, and release episodes on time (McHugh, 2016). The pressure of producing and releasing high quality (and sometimes lengthy) episodes are due to the way listeners consume podcasts.

Podcast consumption is immersive because podcasts are portable. The sound travels with the listener; for example, from earphones, to a car's speakers, to a home speaker. The continuity of content allows the listener to multitask (Morris \& Patterson, 2015): with earphones the listener could be walking, in the car they are driving, at home they could be cooking or cleaning. The near-pervasiveness is most felt when listening to podcasts alone and in personal spaces-a private and intimate experience (Berry, 2016). Listening while shifting environments shows the complexities and modernity of podcast technology.

Traditional radio offers a linear listening experience. Shows are set on a schedule and it is up to the listener to purposefully tune in. Podcasts, on the other hand, are non-linear. Listeners create their own schedule and decide how long to listen for (with the option to tune in episodically or binge the show) (Berry, 2016). They can also control audio playback. Unlike radio, podcast listening is intended to start at the beginning of the episode, with the option of 
“time-shifting” (Berry, 2006; Berry, 2016), meaning playing, pausing, and scrubbing through content. With updated listening applications, Morris and Patterson (2015) suggest time-shifting has evolved into "sonic interactivity." They describe their term as beyond regular audio guidance because of added skills such as jumping through content in increments, adjusting playback speed, and removing silence. Essentially, podcast listeners choose when to listen at a time that is most convenient for them, as opposed to a radio's scheduled shows. Because radio is broadcast through stations, its shows' content is usually designed to appeal to a broader audience than individual podcasts. Podcasts are different in that they are aimed towards niche audiences and listeners can curate a set of shows specific to their own interests.

There are many podcasts to choose from, and audio streaming services make it easy for the listener to see what is available to them. This suggests podcasts are mass marketed but still catered to niche audiences (Morris \& Patterson, 2015; Berry, 2016). Curation is an essential trait when closely interacting with podcasts and utilizing the autonomy offered by them (Berry, 2016). Without it, listeners may lose interest and concentration and tune out (Nyre, 2015). Curation is a personalized experience. Because listeners want to choose how and what to spend their time on, their feed is self-scheduled and self-selected (Morris \& Patterson, 2015; Nyre, 2015). Their chosen podcasts may come from many different genres including narrative storytelling, fiction, investigative, experimental, and talk radio, among many others. McHugh (2016) found a subjective distinction when interviewing audio editors, in that one considered the American narrative to be host-driven and "spoon-fed" (McHugh, 2016, p. 78)-yet is more popular than the European poetic-style antecedent. Therefore, taking this discrepancy into account, this suggests that studying hosts' behaviors are especially important for this host-driven podcast and American podcasts in general. 
Perhaps the defining advantage of podcasting is the autonomy offered throughout on both ends of the communication process. Producing a podcast is an independent venture, both in the de-professionalization of its distribution and by the podcast host. Many creative choices are made by the host alone. As for the listener, many little decisions of who, what, where, when, why, and how must be made in order to get to the feelings of connection and belonging that make podcast listening enjoyable. The next section explores audience satisfaction with other media.

\section{Parasocial Interaction in Radio}

Since Horton and Wohl's foundational article (which looked at PSI in relation to 1950s television), the theory has since been applied to other media forms. These studies contribute a useful foundation for studying PSR and podcasts. As a disclaimer, the evidence found for this examination mostly concentrates on radio. According to the research, identification and perceived similarity are causes of PSI in media audiences.

Identifying with a persona requires noting their values, attitudes, beliefs, and behaviorswhether through stereotyping, what or how they say things, or their actions, and relating them to oneself. The strength of PSI with a persona can be predicted by the ability to identify with them (Horton \& Wohl, 1956; Tian \& Hoffner, 2010; Eyal \& Dailey, 2012). Relatedly, perceived similarity plays a part in the development of PSI.

Perceived similarity can be considered as part of identification, as it is finding what is alike between the persona and someone the audience member knows in real life. Both parasocially and in real-life, putting forth time and commitment leads to an increased connection, especially by the one making the effort (Eyal \& Dailey, 2012). Horton and Wohl described "knowing" personae like friends (p. 216) when characterizing the term. Many have gone on to 
make the same claim, agreeing that how we go about maintaining real-life relationships applies to parasocial ones as well (Tian \& Hoffner, 2010; Eyal \& Dailey, 2012; Rosaen \& Dibble, 2017).

In a way, the two causes are interrelated. One must first identify and compare a persona's characteristics before seeing the perceived similarity between the host and themselves or someone they know. Applying these traits to podcasts show the intricacies of the medium.

\section{Parasocial Traits in Podcasts}

The last section of the literature review revisits and expands on the traits in radio that may facilitate PSRs in audience members, while considering the possibility that unique technical aspects of podcasting may also shape listeners' experiences. I relate the two to develop expectations about the possible traits found in podcasts, which may be precursors to the development of PSRs. This exploration forms the foundation for themes that are coded for in the episodes examined.

\section{Identification}

Audience members of previous media studies, especially those of podcasts, reported feelings of identification that related to their PSRs. Perks and Turner's study (2018) found identification to be a PSI theme in their uses and gratifications (U\&G) study on podcasts. Not only are listeners more interested in the host than the actual content (Piper, 2015; McHugh, 2016), they also found that listeners perceive the host to be "just like them" because of their similar interests (Berry, 2006; Hilmes \& Lindgren, 2016; Lindgren, 2016). Furthermore, Lindgren (2016) specifies in her study that podcasts cater to niches because it takes a specific type of listener to stay engaged, critically think, and make connections - all while following the flow of content. She suggests the connection that loyalty is produced by feeling like listeners know the host personally and because hosts engage with them in a conversational manner. 


\section{Conversation Practices}

Also considered as a U\&G PSI theme (Perks \& Turner, 2018), the ability of a podcast host to sound conversational affects the listening experience. Since podcasts are an audio-only medium, they preserve an unstructured conversation that is not usually seen or felt in other media (Meserko, 2015). Horton and Wohl's 1956 study comments on this “flow of small talk" (p. 217), saying personae give off the impression that they are responding and sustaining a conversation with the audience member, therefore blurring the lines between audiences and media personae.

When the podcast has more than one speaker (whether it be more than one host or a guest on the show), it suddenly feels like a "group" conversation between the speakers and listener. The listener thus feels they are included and are allowed to participate with them (Perks \& Turner, 2018). One audio editor McHugh (2016) interviewed called these bantering back-andforth's by hosts "chumcasts" because they're "talkier" (p. 71) than storytelling narrative-style podcasts. Considering the perceived similarity aspect described earlier, sharing hosts' lives through an ongoing narrative in serialized installments furthers the parasocial bond-as if the listener is checking in on friends (Piper, 2015). Since the podcast chosen for this study has two hosts, I look at how they converse with each other. Instead of plain conversation, sharing personal narratives adds authenticity to a host's voice, creating a feeling of closeness for the listener.

\section{Authenticity}

Meserko (2015) defines authenticity as "an aspirational ideal that informs meditative practices and introspective thought, but also one that informs the often emotional...actions we perform (p. 801)." Not only is it the host's tone of voice, but also their personable, unfiltered language and contemplative self-examination that makes it easier for their listeners to relate to them (Meserko, 2014; Hilmes \& Lindgren, 2016) — or at least the version of themselves they 
want to share. In the initial examination of PSI, Horton and Wohl (1956) noted that personae have contrived public and private images, selecting only parts of each to share with the audience. Comparable to a media figure or business staying "on brand," Horton and Wohl say personae must somewhat adapt to the characteristics being asked of them by the version of themselves the public knows.

Especially with celebrities, Meserko (2014) observed that conversing on a podcast helps speakers convey more of their authentic selves (versus what is seen in the media). He examined the podcast Mental Illness Happy Hour and pinpointed parts of the conversation where the celebrities showed vulnerability when talking about their mental illness struggles. Sharing serious self-disclosures, and even more lighthearted ones, is important for hosts to display, so listeners understand the incorporation of a media figure's private self to their public image.

Adding to authenticity, Perks and Turner (2018) argued that self-disclosure facilitates PSI. When listeners hear self-disclosures from the host, it enhances their feeling of connectedness (Perks \& Turner, 2018) and their ability to identify and cultivate intimacy with them (Meserko, 2014). Although there is a need to be transparent, there is, again, the tricky part of balancing the privacy of one's life from listeners (Piper, 2015). The accumulation of selfdisclosures transforms into an "authentic selfhood." This is the creation of a more authentic image by sharing self-disclosures (Meserko, 2015).

The pursuit of authentic selfhood is developed in real time (Meserko, 2014) through sharing self-contemplations in each episode. Being present during the process of achieving authentic selfhood is also a way to band together the host and their listeners. When studying selfhelp podcasts, Meserko (2014) found that speakers share intimate thoughts because they believe their audience is likeminded. It is as if it is a way of "acting together" (p. 463) to overcome 
shared debilitations. It's perceived as teamwork from both sides. The host makes it easier for the audience to relate to their personal narrative by sounding personable and authentic, thus increasing intimacy (Lindgren, 2016).

\section{Intimacy}

Podcasts listeners report effects of intimacy, which leads to greater authenticity and conversation practices than radio. When hosts share personal topics, as discussed above, it is considered a sign of intimacy (Perks \& Turner, 2018). With earphones, the host's voice is literally in the listener's ear and speaking only to them (Hilmes \& Lindgren, 2016). For example, an interviewee of MacDougall's study (2011) commented: "I can hear him take breaths between sentences. He's a great speaker. Like he's next to me” (p. 726). Through mediated isolation (escapism) and hyper-intimacy (deep engagement), listeners are transported to an internalized environment, which Berry (2016) suggests may lead to "parasocial phenomena" (p. 13). To use Horton and Wohl's (1956) coined term, the "illusion of intimacy" (p. 217) is amplified in podcasts enough for the listener to feel like their efforts are being reciprocated, and in turn, allow the hosts to influence how they interpret the content.

Although it is just as important of a parasocial trait as the others, this study did not look for intimacy in its research methods. Intimacy is a subjective feeling, observed in firsthand accounts and audience data. This study focused on the hosts of a podcast (instead of the listener) and used secondary data in the form of transcripts. Therefore, examining intimacy was outside the scope of research.

\section{Methodology}

I conducted a deductive thematic analysis using episodes of the podcast Stuff You Should Know (SYSK). The research method chosen for this study was selected for its utility to 
extensively focus on one artifact and uncover and connect hidden themes, especially of exploratory, initial research or less studied topics. SYSK, is a talk radio podcast dedicated to educating listeners about various topics. It consists of two male hosts, Chuck Bryant and Josh Clark, both writers for How Stuff Works, the organization that produces SYSK, and who are also hosts of other podcasts under the same organization. I chose this podcast because there was already initial evidence of parasocial implications through listener loyalty. It has existed for eleven years, won multiple audio awards, and toured North America. Additionally, SYSK has a segment dedicated to "listener mail" in which listeners write in to add to the conversation of a past episode and/or express how the show has influenced their life. I chose to focus on only one podcast to better elaborate on how the repetitive exposure to the same hosts (and their habits) may cultivate PSR.

I gathered five episodes to draw evidence from - a total of almost three hours of content. I listened to each episode and examined transcripts. I chose episodes that had existing transcriptions, supplied by the podcast's website. I then coded for themes that reflect the possibility that listeners will develop PSR with the hosts. See the references page for a full list of the episodes used.

The themes I coded for are based on the podcast traits from the last section of the literature review: identification, conversation practices, and authenticity. As explored in their respective sections above, each trait may contribute to the likelihood of the development of PSR. By coding for these themes, I addressed the following research question: "How are parasocial relationships cultivated by podcast hosts?"

Identification is when the listener relates to the podcast hosts' values, attitudes, beliefs, and behaviors. To capture the ways in which podcast hosts cultivate identification, I coded for 
several related elements (see Table 1). When hosts directly state their characteristics and talk about their personal life (whether or not relating to the topic at hand), listeners apply the characteristics onto themselves (Meserko, 2014; Meserko, 2015). Sometimes, hosts give their listeners advice (Meserko, 2014; Merserko, 2015; Collins, 2018). Since co-presence is at play, hosts give advice to their mass audience using the second-person point-of-view (Montgomery, 1986), and listeners are willing to receive it. Another code, "expressives," coined by Searle (as cited by Montgomery, 1986), are uninvited remarks made by the speaker that implies their attitudes or feelings towards a subject. In the case of this podcast, the hosts voice their opinion on the content. Listeners may naturally have similar reactions as the host, thus identifying with them. Altogether, if listeners perceive hosts to be similar to them, they may likely find ways to relate to them as well. Table 1 provides further detail on these categories. 
Table 1: Definitions of Identification Themes and Codes

\begin{tabular}{|l|l|l|}
\hline \multicolumn{1}{|c|}{ Identification Code } & \multicolumn{1}{|c|}{ Definition } & \multicolumn{1}{|c|}{ Example } \\
\hline Description of Personal Characteristics & $\begin{array}{l}\text { Explicitly describing characteristics about } \\
\text { themselves or the other host. }\end{array}$ & $\begin{array}{l}\text { Bryant: I hate mimes. I actually don't hate mimes, } \\
\text { it's just a cliché to hate mimes. Mimes are cool. } \\
\text { (Ep. 127) }\end{array}$ \\
\hline Self-Disclosure of Personal Life & $\begin{array}{l}\text { Revealing details of their personal life in the } \\
\text { form of stories and recalling events. }\end{array}$ & $\begin{array}{l}\text { Clark: When I was in elementary school, my mom } \\
\text { made me an upside-down clown costume. So, from } \\
\text { my legs, my shoulders were sticking up. (Ep. 257) }\end{array}$ \\
\hline Personally Relating to Content & $\begin{array}{l}\text { Using examples from their personal life to } \\
\text { connect to the podcast's content. }\end{array}$ & $\begin{array}{l}\text { Clark: My first father-in-law got like shunts put in. } \\
\text { He was smoking and golfing a couple days later. He } \\
\text { just wouldn't stop. Heart shunts I should say. Cardiac } \\
\text { shunts! (p. 105) }\end{array}$ \\
\hline Advice Giving & $\begin{array}{l}\text { Providing solutions or suggestions in/directly } \\
\text { applicable to the listener. }\end{array}$ & $\begin{array}{l}\text { Bryant: But you're going to have a good head start } \\
\text { on figuring out a good place to start, and then just } \\
\text { observe. Go from there, do the eyeball test, as they } \\
\text { say, and if things seem to be working, then great. } \\
\text { (Ep. 700) }\end{array}$ \\
\hline Expressives & $\begin{array}{l}\text { Side comments to convey hosts' attitudes or } \\
\text { feelings towards the content presented. Their } \\
\text { opinion towards the content. }\end{array}$ & $\begin{array}{l}\text { Bryant: All I know is the dog was put to sleep, } \\
\text { which is really upsetting to me. } \\
\text { Clark: That is upsetting, especially if it was trying to } \\
\text { rescue her. (Ep. 105) }\end{array}$ \\
\hline
\end{tabular}


Conversation practices relate to the conversation quality produced by podcast hosts. Since there are two hosts weighing in on the topic of every episode of SYSK, as Perks and Turner (2018) found, the listener may feel like there is "space for them in the conversation" (p. 109). The codes chosen for this theme (see Table 2) are naturally-occurring elements in conversations, with the most conventional cases being the use of examples to expand on the content and the use of expressions and colloquialisms (Tolson, 2010). The other conversation codes, social deixis, spatial deixis, and speaker change, are more often analyzed in media settings. Social deixis is when the speaker directly addresses the audience (Meserko, 2014, Tolson, 2010, Montgomery, 1986), thus creating a co-presence with them in real time in a virtual space. As for spatial deixis, it can be seen as "erasing a sense of distance between speaker and audience - assuming a common visual field thereby implies a form of co-presence" (Montgomery, 1986, p. 429). So, in podcasts, the hosts may refer to activity happening within their studio during the time of recording. Lastly, I code for speaker change (Montgomery, 1986), or how the hosts transition between each other. These interactions show how hosts create a conversational feel so that listeners are more engaged with the content, and feel they are participating too. 
Table 2: Definitions of Conversational Themes and Codes

\begin{tabular}{|l|l|l|}
\hline \multicolumn{1}{|c|}{ Conversation Practices Code } & \multicolumn{1}{|c|}{ Definition } & \multicolumn{1}{|c|}{ Example } \\
\hline $\begin{array}{l}\text { Expanding Understanding Through } \\
\text { Examples }\end{array}$ & $\begin{array}{l}\text { Using examples of modern society or } \\
\text { hypothetical situations to expand on the } \\
\text { podcast's content. Can also be expressed in } \\
\text { the form of dialogue. }\end{array}$ & $\begin{array}{l}\text { Bryant: During peacetime, Josh, they would have } \\
\text { these tournaments and it was big - it was like the } \\
\text { NFL football of the day. } \\
\text { Clark: It was like the NFL and World Cup put } \\
\text { together. (Ep. 257) }\end{array}$ \\
\hline Expressions & Easily understood popular expressions. & $\begin{array}{l}\text { Bryant: Or I get the feeling from page to squire } \\
\text { was a little more learning, but then from squire to } \\
\text { knight was when the rubber meets the road. } \\
\text { (Ep. 257) }\end{array}$ \\
\hline Social Deixis & $\begin{array}{l}\text { Depicting and directly addressing the } \\
\text { audience as a singular listener. Simulating co- } \\
\text { presence by addressing the mass audience in } \\
\text { second-person. }\end{array}$ & $\begin{array}{l}\text { Bryant: I'm not going to tell you how to parent, } \\
\text { but I think it's a good idea to have a good, open, } \\
\text { honest line of dialogue with your kid about } \\
\text { homeschooling first. (Ep. 700) }\end{array}$ \\
to their physical environment. & $\begin{array}{l}\text { Bryant: Okay, we're back, and we have a third } \\
\text { person in the studio. } \\
\text { Clark: Stuff Mom Never Told You's Cristen } \\
\text { Conger. (Ep. 700) }\end{array}$ \\
\hline Speaker Change & $\begin{array}{l}\text { How the hosts interact with each other when } \\
\text { providing information. }\end{array}$ & $\begin{array}{l}\text { Bryant: Oh, shut up. You've had McDonald's in } \\
\text { your day, pal, Twinkie Man, Twinkie the Kid. } \\
\text { Clark: Let's talk about Twinkie the Kid. Nice } \\
\text { segway. } \\
\text { Bryant: I just totally shocked you with that. } \\
\text { (Ep. 127) }\end{array}$ \\
\hline
\end{tabular}


Finally, I code for several markers of authenticity (see Table 3). According to Meserko, (2015), authenticity triggers introspection and emotion in listeners. He also notes that since we have a sense of our own individual identity, we can therefore observe it in others. It is understood that because the podcast medium is "de-professionalized," so are the hosts of a majority of its shows, including SYSK. As “ordinary experts" (Tolson, 2010), Bryant and Clark are transparent with their imperfections. Their listeners may notice this through codes of uncertainty, corrections (concerning the content), and contemplative self-examination (Meserko, 2014; Meserko, 2015). They also set themselves apart from other podcasts by adding personality and a distinct dynamic when conversing with each other. This can be seen through exclamations, off-topic conversations (Collins, 2018) and wit. The last-mentioned code demonstrates speech as a "blend of humour and intellectualism" (Drew, 2017, p. 208). To observe authenticity, I code for how the hosts naturally interact with and react to the content. Listeners can then observe how hosts' personalities merge with straightforward facts. 
Table 3: Definitions of Authenticity Themes and Codes

\begin{tabular}{|c|c|c|}
\hline Authenticity Code & Definition & Example \\
\hline Exclamations & $\begin{array}{l}\text { Comments in which the host sounds stunned; } \\
\text { incredulous responses to content presented. }\end{array}$ & $\begin{array}{l}\text { Clark: Wow. } \\
\text { Bryant: Looks a lot better. Doesn't he? } \\
\text { Clark: Wow. Quite the difference! Yeah. } \\
\text { (Ep. 105) }\end{array}$ \\
\hline Uncertainty & $\begin{array}{l}\text { Explicitly stating they are unsure of the } \\
\text { content they presented, or the content may } \\
\text { not be accurate. }\end{array}$ & $\begin{array}{l}\text { Bryant: Well, I don't know if it was aliens } \\
\text { necessarily, but I think it was probably - what I } \\
\text { think, it was some kind of Army experimentation, } \\
\text { radiation, maybe bombs, something like that. } \\
\text { (Ep. 99) }\end{array}$ \\
\hline Corrections & $\begin{array}{l}\text { Admitting to mistakes or inaccuracies of the } \\
\text { content presented. Also includes disclaimers, } \\
\text { meaning corrections made before the } \\
\text { mistakes happen. }\end{array}$ & $\begin{array}{l}\text { Bryant: And as of the writing on this article, we } \\
\text { actually need to update this article that there were } \\
\text { no full transplants, but there have been since then. } \\
\text { (Ep. 105) }\end{array}$ \\
\hline Contemplative Self-Examination & $\begin{array}{l}\text { Demonstrates hosts' introspection about their } \\
\text { thought process in real time. }\end{array}$ & $\begin{array}{l}\text { Bryant: I think I'm a much more visual learner. } \\
\text { Clark: Yeah, I get it when I see it too, but it } \\
\text { doesn't-I don't get it until I write it myself. } \\
\text { Bryant: Actually, I'm a combination between } \\
\text { visual and kinesthetic, because kinesthetic is really } \\
\text { being involved... (Ep. 700) }\end{array}$ \\
\hline Off-Topic Conversation & Digression from the main topic. & $\begin{array}{l}\text { Clark: He was married as The Edge though. } \\
\text { Bryant: Was he really? } \\
\text { Clark: Yeah, I remember when he got married, I } \\
\text { don't know if, like, in the ceremony, but in all of } \\
\text { the spreads it was like, The Edge and Mrs. Edge. } \\
\text { (Ep. 257) }\end{array}$ \\
\hline Wit & $\begin{array}{l}\text { Tone and/or speech change between one or } \\
\text { both hosts to portray intellect through humor } \\
\text { or lightheartedness; sarcasm; unplanned } \\
\text { banter. }\end{array}$ & $\begin{array}{l}\text { Bryant: Carbon-what kind-carbon steel? } \\
\text { Clark: Yeah, but usually light on the carbon steel. } \\
\text { Bryant: Oh, light on the carbon steel? } \\
\text { Clark: Um-hum. } \\
\text { Bryant: Heavy on the ketchup. (Ep. 257) }\end{array}$ \\
\hline
\end{tabular}




\section{Results}

By conducting a thematic analysis on SYSK, I discovered five repetitive circumstances throughout the episodes examined that reflect how the parasocial codes and themes work together: the use of introductions to ease into the episode's topic, the hosts' creations of hypothetical situations and dialogue to expand on content, how the hosts interact with each other when transitioning through sub-topics, how the hosts give advice to their listeners, and how they conduct their listener mail segment. My compiled codes and themes incorporated these patterns to exemplify how they work together to further parasocial relationships.

\section{Identification}

The most prominent codes found amongst the episodes examined for the identification theme included self-disclosure, advice giving, expressives, and descriptions of personal characteristics. First off, self-disclosure is mostly seen in the introductions of each episode. The hosts ease into the main topic. Especially in the homeschooling episode (Ep. 700), Clark asks Bryant questions about his parents' occupations, already knowing that they were both educators. It was Clark's way of shifting into the subject of homeschooling. Also, at the end of podcast episodes when the hosts answer listener mail, listeners sometimes ask them personal questions (about their relationships, for example) (Ep. 257); the hosts sincerely answer them. These private self-disclosures may have otherwise never been revealed in the podcast.

The advice giving pattern noticeably includes the advice giving code. Again, referring to the homeschooling episode (Ep. 700), the whole episode's purpose was to teach and help the listener figure out if homeschooling was right for their family. They ask a series of questions directly to the listener of how they want/should go about starting the homeschooling process. Then, they continue on to tell the listener about teaching method options. 
Expressives are scattered throughout each episode, but it most often occurs in the listener mail segment. The hosts share that they appreciate the messages they get from listeners and respond to all types of messages. Examples include thanking them for writing in, indicating they love to be corrected (Ep. 105), and enjoy when listeners share firsthand accounts concerning past topics (Ep. 99). Expressives display the hosts' personal opinions about the content.

Lastly, the most frequent code that came up was the description of personal characteristics. These descriptions are either of themselves or each other. They also occur throughout each episode. A distinct example of this was in the knights' episode (Ep. 257), in which they apply their characteristics to the content or how they would interact in hypothetical situations. They first explained the different roles of a squire, then directly asked the listeners to guess which type of squire Clark is most like. The two ultimately decide Bryant would be a squire of the pantry, and Clark, a squire of the wines. Collectively, they passed the point of simply teaching the listener, to having the listener work through the new information they were given.

\section{Conversation Practices}

The codes used to elaborate on the conversation practices theme included dialogue, speaker change, and both spatial and social deixis. The theme included the expanding understanding through examples code. Since the hosts utilize dialogue as a different way to explain concepts, dialogue is therefore under this code. The section above included a brief discussion about one way the hosts used dialogue. Now, instead of the dialogue portraying the hosts' characteristics, the results concentrated on how they use modern-day speech to explain concepts - still through hypothetical situations. For instance, again in the knights' episode (Ep. 257), they talk about a king's law of owning land. 
Clark: I guess he conquered, or he just said, "Hey, this is mine. This is my land."

Bryant: And not, "This land is your land."

Clark: Here's some land.

Bryant: "It's yours."

Clark: But you owe me big time for that land.

Obviously, they are not in the king's time period, but like this example, they act out imaginary examples to help demonstrate intricate concepts. They bring historical figures, thought processes, and theories to life, by personifying them in hypothetical situations.

Transitioning between hosts and sub-topics may be confusing for the listener-especially with a loosely structured podcast like SYSK. Usually the hosts' speaker change is either clearly and properly set up or flows smoothly into the next subtopics. When this is not the case, sometimes they "check in" with each other before moving on. This most often occurred in the episode about bizarre deaths (Ep. 99), either deciding on the spot who speaks about which subtopic or asking the other for permission, such as: "Can I go on? Can I do this next one?" Despite these spur-of-the-moment transitions, the hosts are still able to get their points across.

Spatial deixis was only particularly seen in the introduction section of each episode. The code is defined as a seemingly "physical" co-presence, because the hosts describe the space they are currently in to the listener. Using the twinkies episode's (Ep. 127) introduction to elaborate, the hosts start off by mentioning their sound producer, Jeri. She is always present with them in the recording booth, but never talks (an ongoing joke within the podcast). Therefore, they imply the space and time they're in (recording booth) and the people present in the room.

As for social deixis, its ability to create the feeling of co-presence for the listener was found when the hosts gave advice, as well as in their listener mail segment. Both patterns addressed the listener in second-person, thus speaking to their mass audience as if they have just one listener. Once again, on the subject of squires (Ep. 257), the hosts "play a game" with the 
listener. Although he refers to a mass audience, Bryant nevertheless understands he's speaking to individual listeners: "I wish we could do instant voting, but say in your heads people out there listening, what you think Josh would have been."

Another example comes from the homeschooling episode (Ep. 700), when explaining the homeschooling process. They directly ask questions like, "Why do you want to do this? Can you afford it? Are you qualified to teach?" In this case, social deixis displays that the hosts are aware they sound like they are only speaking to one listener.

\section{Authenticity}

Finally, the most notable codes for the authenticity theme were uncertainty, selfexamination, corrections, going off-topic, and wit. To begin, I have also already discussed that the hosts enjoy being corrected by their listeners, but the occurrence also counts towards the correction code. They even admit they are wrong. In the episode about face transplants (Ep. 105), they had written an article on the podcast's blog about the same topic, and during the episode they expressed that they needed to go back to change inaccuracies. As stated in the code's definition (see Table 3), the correction code also includes disclaimers. In the episode about bizarre deaths (Ep. 99), they note are aware it is a serious topic and are careful not to be too comical or lighthearted, as they usually are.

As indicated before, the hosts occasionally struggle with transitioning into the next subtopic. The uncertainty of what to speak about next is visibly there. Uncertainty may also happen when hosts are going through contemplative self-examination. In one instance, Clark struggles on how to start off the topic of knights (Ep. 257). He explained his thought process out loud, "I was looking all over for something to lead in with this. I considered talking about the paladin class character I created when I played Dungeons and Dragons as a kid.” He goes on, 
deliberating on subjects concerning sports, news, and politics. These instances can only be coded for, again, when the hosts think out loud; they should not be inferred.

Their comical personalities are especially seen when they go off-topic. This banter is largely seen in the introductions because again, the hosts ease into the topic. Using the example from the spatial deixis code, because Jeri doesn't talk, they compare her to a mime, then go off on a quick tangent about mimes. Even though it was most commonly seen in the introduction section, their tangents are frequent throughout the episode. For example, talking about an attractive bus driver, when the episode is supposed to be about twinkies (Ep. 127).

Their tangents usually include wit. They add jokes and witty comebacks towards each other in between the educational information. An instance of this occurs when Clark reveals the most twinkies ever eaten (Ep. 127).

Clark: So, Chuck, most Twinkies ever eaten by a person; you want to guess who it is? Bryant: Uh, Jeri, our producer.

Clark: She's close.

Bryant: Okay.

Clark: She's very close. Actually, there is a guy who is eighty-nine and I believe he lives in Indiana and his name is Louis Browning. He's been eating a twinkie a day since 1941.

The witty exchange flows into the fact. These types of responses are quick, abrupt, and improvised. This exchange is also a good example of how they tease Jeri, as mentioned earlier.

\section{Discussion}

Five implications were made out of the findings. Each theme was connected with at least one other theme to indicate instances of PSI for a listener. The implications also build upon each other and suggest the ways in which PSR is cultivated.

The first implication deals with the description of personal characteristics code of the identification theme and spatial deixis from the conversation practices theme. The hosts describe 
the current place they're in and the listener perceives the moment is happening in real time (which is never the case since podcasts are pre-recorded and edited before being posted). Then, as the hosts talk with their off-topic, witty banter, the listener gets to know both the comical and personal sides of the hosts. The interaction of these two themes creates a feeling of both physical and emotional closeness for the listener.

Once the listener gets to know the hosts, understanding their humor and how they interact with each other (still examined here with the description of personal characteristics code), may help the listener to understand the content better, especially through dialogue. Dialogue is considered as part of the expanding understanding through examples code of the conversation practices theme. As mentioned before, the hosts' use of dialogue has two functions: portraying the hosts' characteristics through the choices they make in hypothetical situations, as well as personifying thought processes and concepts for better understanding. When teaching, applying concepts after learning about them is the next step to fully comprehending the material. Therefore, when these themes interact, the listener translates it as a courtesy of the hosts to help them fully grasp the otherwise complex topics.

As discussed before, since the hosts' dialogue and speaker changes (a code from the conversation practices theme) are made in real time, transitions from one topic to the next can get jumbled. Coupling speaker change with the uncertainty code of the authenticity theme still works to the hosts' advantage. This is because the hosts still get their job done. They are flexible with however the episode navigates and are comfortable speaking about any part of the topic. The listener thus perceives them as more credible, authoritative, and trustworthy because they still manage to educate the listener by the end of the episode. 
The listener's trust towards the hosts is also seen when advice giving and social deixis codes are present, from the themes of identification and conversation practices, respectively. Social deixis strengthens the co-presence of the listener, and when the hosts give advice, it gives the impression that they care for the individual listener. In a friend-like fashion, they form a call to action for the listener's wellbeing. When the listener senses this, it increases their trust towards the hosts.

The listener mail segment of SYSK consistently incorporates all three themes. Mostly, it uses social deixis, similar to giving the listener advice. What is different about this segment is the hosts reply back to the parasocial conversation. Similar exchanges happen whenever a media figure acknowledges the presence of a singular audience member; this occurrence is not exclusive to SYSK or podcasts in general. Even though the hosts only respond to listeners that send them mail, it still makes the individual listener feel heard and included. It demonstrates that the hosts are putting in effort to participate in the listener's PSR.

These implications relate back to Horton and Wohl's (1956) notion of the audience member thinking they know the personae like a friend. The findings suggest a number of implications. That the listener can get to know hosts and even feeling physically closer to them. The hosts make sure that listeners better understand what they're talking about, as well as give them advice, both of which depict the hosts as caring people and increase the listener's trust towards them. Lastly, the hosts can reciprocate the time and effort the listener puts into the podcast by responding back to their mass audience. The brief acknowledgment that the hosts know the listener is tuned in, makes the commitment and loyalty all worthwhile for the listener. The repetition of the interaction of these parasocial themes accumulate and suggest the cultivation of PSR. 


\section{Limitations and Directions for Future Research}

The limitations of this study were mostly due to time constraints. Five episodes of only one podcast were examined. These episodes were chosen because the transcripts were already available on the SYSK website. Future research should use a random sample of episodes or use episodes that have plenty of evidence of the themes. Additionally, earlier episodes were examined, so future research could examine more recent episodes, possibly to compare the consistency and growth of the hosts and themes. Future research should also explore PSI/PSR across multiple podcasts to find consistencies and create themes that work for most, if not all, podcasts.

\section{Conclusion}

Although there have been studies about parasocial relationships in other media, and parasocial implications in podcast studies, there is little research concerning parasocial phenomena and podcasts. Therefore, I performed a thematic analysis to uncover how certain content characteristics might relate to the development of PSR in listeners, through podcast hosts.

Podcasts are a part of the shift in the ever-evolving media and technology landscape, in which media now have self-autonomous abilities for the consumer. At the same time, podcasts are becoming increasingly popular. With these two cases in mind, PSR plays an important part in the development of podcast choices. As listeners are able to focus their consumption on more specific interests, they may find that their PSR with the podcast, and especially its host, strengthens. 
As this study demonstrated, PSRs with podcast hosts may cultivate the same way real-life relationships do. As social relationships are important to us, with the new self-autonomous and personalized advances in our media and technology landscape, we can have the same intimate relationships with media figures. Again, because of this advanced personalization PSRs may quite possibly be stronger than ever before. Although, if this is the case, it is important to find the ways in which media figures (in this case, podcast hosts) cultivate PSR in listeners, as critical questions of replacing real relationships with parasocial ones may arise. 


\section{References}

Berry, R. (2006). Will the iPod kill the radio star? Profiling podcasting as radio. Convergence, 12(2), 143-162.

Berry, R. (2016). Podcasting: Considering the evolution of the medium and its association with the word 'radio'. Radio Journal: International Studies in Broadcast \& Audio Media, 14(1), 7-22.

Bryant, C., \& Clark, J. (Hosts). (2009, April 2). Episode 99: Bizarre ways to die [Audio podcast].

Bryant, C., \& Clark, J. (Hosts). (2009, April 23). Episode 105: How face transplants work [Audio podcast].

Bryant, C., \& Clark, J. (Hosts). (2009, July 9). Episodes 127: How twinkies work [Audio podcast].

Bryant, C., \& Clark, J. (Hosts). (2010, October 7). Episode 257: How knights work [Audio podcast].

Bryant, C., \& Clark, J. (Hosts). (2014, December 11). Episode 700: How twinkies work [Audio podcast].

Collins, K. (2018). Comedian hosts and the demotic turn. Podcasting: New Aural Cultures and Digital Media, (1)12, 227-250.

Drew, C. (2017). Educational podcasts: A genre analysis. E-Learning and Digital Media, 14(4), 201-211).

Eyal, K., \& Dailey, R. M. (2012). Examining relational maintenance in parasocial relationships. Mass Communication and Society, 15(5), 758-781. 
Hilmes, M., \& Lindgren, M. (2016). Podcast review and criticism: A forum. Radio Journal: International Studies in Broadcast \& Audio Media, 14(1), 83-89.

Horton, D., \& Richard Wohl, R. (1956). Mass communication and para-social interaction: Observations on intimacy at a distance. Psychiatry, 19(3), 215-229.

Lindgren, M. (2016). Personal narrative journalism and podcasting. Radio Journal: International Studies in Broadcast \& Audio Media, 14(1), 23-41.

MacDougall, R. C. (2011). Podcasting and political life. American Behavioral Scientist, 55(6), 714-732.

Markman, K. M., \& Sawyer, C. E. (2014). Why pod? Further explorations of the motivations for independent podcasting. Journal of Radio \& Audio Media, 21(1), 20-35.

McClung, S., \& Johnson, K. (2010). Examining the motives of podcast users. Journal of Radio \& Audio Media, 17(1), 82-95.

McHugh, S. (2016). How podcasting is changing the audio storytelling genre. Radio Journal: International Studies in Broadcast \& Audio Media, 14(1), 65-82.

Meserko, V. M. (2014). Going mental: Podcasting, authenticity, and artist-fan identification on Paul Gilmartin's Mental Illness Happy Hour. Journal of Broadcasting \& Electronic Media, 58(3), 456-469.

Meserko, V. M. (2015). The pursuit of authenticity on Marc Maron's WTF podcast. Continuum, $29(6), 796-810$.

Montgomery, M. (1986). DJ talk. Media, Culture \& Society, 8(4), 421-440.

Morris, J. W., \& Patterson, E. (2015). Podcasting and its apps: Software, sound, and the interfaces of digital audio. Journal of Radio \& Audio Media, 22(2), 220-230. 
Nyre, L. (2015). Urban headphone listening and the situational fit of music, radio and podcasting. Journal of Radio \& Audio Media, 22(2), 279-298.

Perks, L.G. \& Turner, J.S. (2018). Podcasts and productivity: A qualitative uses and gratifications study. Mass Communications and Society, 22(), 96-116.

Tian, Q., \& Hoffner, C. A. (2010). Parasocial interaction with liked, neutral, and disliked characters on a popular TV series. Mass Communication and Society, 13(3), 250-269.s

Tolson, A. (2010). A new authenticity? Communicative practices on YouTube. Critical Discourse Studies, 7(4), 277-289. 